PROCEEDINGS OF THE

AMERICAN MATHEMATICAL SOCIETY

Volume 134, Number 1, Pages 229-234

S 0002-9939(05)07973-6

Article electronically published on June 13, 2005

\title{
AN ELEMENTARY PROOF FOR A CHARACTERIZATION OF *-ISOMORPHISMS
}

\author{
S. H. KULKARNI, M. T. NAIR, AND M. N. N. NAMBOODIRI \\ (Communicated by Joseph A. Ball)
}

\begin{abstract}
We give an elementary proof of a result which characterizes onto *-isomorphisms of the algebra $B L(H)$ of all the bounded linear operators on a Hilbert space $H$. A known proof of this result (Arveson, 1976) relies on the theory of irreducible representations of $C^{*}$-algebras, whereas the proof given by us is based on elementary properties of operators on a Hilbert space which can be found in any introductory text on Functional Analysis.
\end{abstract}

\section{INTRODUCTION}

The aim of this note is to give an elementary proof of the following theorem that characterizes onto *-isomorphisms of the algebra $B L(H)$ of all the bounded linear operators on a Hilbert space $H$.

Theorem 1.1. Let $H$ and $K$ be Hilbert spaces and let $\Phi$ be $a *$-isomorphism from $B L(H)$ onto $B L(K)$. Then there exists a unitary operator $U: H \rightarrow K$ such that $\Phi(T)=U T U^{*}$ for all $T \in B L(H)$.

The classical proof of this theorem relies on the theory of irreducible representations of $C^{*}$-algebras (see, for example, [1]). Our proof uses only very basic properties of the operators between Hilbert spaces, which can be found in any introductory text on Functional Analysis, for example 22 or [3. Some of these properties are also mentioned explicitly at the beginning of the next section. In particular, we do not use any concept involving the spectrum of an operator. This elementary nature of our proof has an added advantage, namely that it works for both real as well as complex Hilbert spaces, whereas in the classical literature, this theorem is stated and proved only for complex Hilbert spaces. Also, the classical version of the theorem stated above does not say anything about the uniqueness of the unitary operator $U$. On the other hand, we have proved by elementary methods that the operator $U$ is unique up to a scalar multiple of absolute value 1 . We thank Professor B. V. Limaye, I. I. T. Bombay, for drawing our attention to this question of uniqueness and also for several other useful suggestions.

Received by the editors August 12, 2004 and, in revised form, August 27, 2004

2000 Mathematics Subject Classification. Primary 47L10; Secondary 47L30.

Key words and phrases. ${ }^{*}$-isomorphism, unitary operator, orthogonal projection.

(C)2005 American Mathematical Society Reverts to public domain 28 years from publication 


\section{MAIN RESUlT}

Let $H, K$ be Hilbert spaces. We denote by $B L(H, K)$ the set of all bounded linear maps from $H$ to $K$, and $B L(H, H)$ will be denoted by $B L(H)$. For $T \in B L(H, K)$, $T^{*}$ will denote the adjoint of $T, N(T)$, the null space of $T, R(T)$, the range of $T$ and $\operatorname{Rank}(T)$, the dimension of $R(T)$.

In what follows, we shall make repeated use of some well-known facts about orthogonal projections. For a ready reference, we collect those facts in the following theorem. Proofs can be found in any introductory text on Functional Analysis (e.g., [2, 3]).

Theorem 2.1. Let $H$ be a Hilbert space.

(1) Let $P \in B L(H)$ be a projection, that is, $P^{2}=P$. Then $P$ is orthogonal (that is, $R(P) \perp N(P)) \Leftrightarrow P^{*}=P$ (that is, $P$ is self adjoint).

(2) Let $P, Q$ be orthogonal projections in $B L(H)$. Then:

(a) $R(P) \subseteq R(Q) \Leftrightarrow P Q=P=Q P$.

(b) $R(P) \perp R(Q) \Leftrightarrow P Q=0=Q P$.

In addition to the above, we shall need some properties of operators and projections of rank one. We prove these in the following proposition.

Proposition 2.2. Let $H$ be a Hilbert space. For $x, y \in H$ define the operator $T_{x, y}$ on $H$ by

$$
T_{x, y}(u)=\langle u, y\rangle x, \quad u \in H,
$$

and let $P_{x}:=T_{x, x}$. Then the following statements hold:

(1) For $x, y \in H, T_{x, y} \in B L(H)$ and $\operatorname{Rank}\left(T_{x, y}\right)=1$.

(2) For $x, y \in H$, and scalars $\alpha, \beta, T_{\alpha x, \beta y}=\alpha \beta T_{x, y}$. In particular, $P_{\lambda x}=P_{x}$ for all scalars $\lambda$ with $|\lambda|=1$.

(3) If $x \in H$ with $\|x\|=1$, then $P_{x}$ is an orthogonal projection of rank 1 .

(4) If $P \in B L(H)$ is an orthogonal projection of rank 1 , then there exists $x \in H$ with $\|x\|=1$ such that $P=P_{x}$.

(5) For $x, y \in H$ with $\|x\|=1=\|y\|$,

$$
\langle x, y\rangle=0 \Leftrightarrow P_{x} P_{y}=0=P_{y} P_{x} .
$$

(6) For $x, y, z \in H, T_{x, z} T_{y, x}=\langle y, z\rangle P_{x}$.

(7) For $x, y \in H,\left(T_{x, y}\right)^{*}=T_{y, x}$.

(8) Let $x, y, z \in H$ with $\|x\|=1=\|y\|$. Then

$$
T_{x, y} P_{z}= \begin{cases}0, & \text { if }\langle z, y\rangle=0 \\ T_{x, y}, & \text { if } z=y\end{cases}
$$

Also

$$
P_{x} T_{x, y}=T_{x, y}, \quad T_{x, y} T_{y, z}=T_{x, z} .
$$

In particular, $T_{x, y} T_{y, x}=P_{x}$.

(9) Let $T \in B L(H)$ be of rank 1 . Then there exist $x, y \in H$ with $\|x\|=1$ such that $T=T_{x, y}$. In fact, $x$ can be chosen as any element of norm 1 in $R(T)$ and in that case $y=T^{*}(x)$.

Proof. Results in (1) and (2) involve routine verification.

(3) Let $x \in H$ with $\|x\|=1$. Then $P_{x}(x)=x$. Hence for all $u \in H$,

$$
P_{x}^{2}(u)=P_{x}(\langle u, x\rangle x)=\langle u, x\rangle x=P_{x}(u) .
$$


Thus $P_{x}$ is a projection. Also $R\left(P_{x}\right)=\operatorname{span}(\{x\})$. Hence, $\operatorname{Rank}\left(P_{x}\right)=1$. Next, for $u \in H, u \in N\left(P_{x}\right) \Leftrightarrow\langle u, x\rangle x=0$. This shows that $N\left(P_{x}\right) \perp R\left(P_{x}\right)$.

(4) Suppose $P$ is an orthogonal projection of rank 1 . Then $P^{*}=P$ by Theorem 2.1 and there exists $x \in H$ with $\|x\|=1$ such that $R(P)=\operatorname{span}(\{x\})$. Let $u \in H$. Then

$$
P(u)=\langle P(u), x\rangle x=\left\langle u, P^{*}(x)\right\rangle x=\langle u, P(x)\rangle x=\langle u, x\rangle x=P_{x}(u) .
$$

Thus $P=P_{x}$.

(5) For $x, y \in H$ with $\|x\|=1=\|y\|$,

$$
\langle x, y\rangle=0 \Leftrightarrow R\left(P_{x}\right) \perp R\left(P_{y}\right) \Leftrightarrow P_{x} P_{y}=0=P_{y} P_{x} .
$$

(6) For $x, y, z, u \in H$,

$$
T_{x, z} T_{y, x}(u)=T_{x, z}(\langle u, x\rangle y)=\langle u, x\rangle T_{x, z}(y)=\langle u, x\rangle\langle y, z\rangle x=\langle y, z\rangle P_{x}(u) .
$$

(7) For $x, y, u, v \in H$,

$$
\begin{aligned}
\left\langle u,\left(T_{x, y}\right)^{*}(v)\right\rangle & =\left\langle T_{x, y}(u), v\right\rangle=\langle u, y\rangle\langle x, v\rangle \\
& =\langle u, \overline{\langle x, v\rangle} y\rangle=\langle u,\langle v, x\rangle y\rangle=\left\langle u, T_{y, x}(v)\right\rangle .
\end{aligned}
$$

(8) For $x, y \in H$ with $\|x\|=1=\|y\|$, and $z, u \in H$,

$$
T_{x, y} P_{z}(u)=T_{x, y}(\langle u, z\rangle z)=\langle u, z\rangle T_{x, y}(z)=\langle u, z\rangle\langle z, y\rangle x .
$$

We observe that

$$
\langle u, z\rangle\langle z, y\rangle x= \begin{cases}0, & \text { if }\langle z, y\rangle=0 \\ \langle u, y\rangle x, & \text { if } z=y\end{cases}
$$

Other assertions can be proved in a similar way.

(9) Let $x \in R(T)$ with $\|x\|=1$ and $y=T^{*}(x)$. Since $\operatorname{Rank}(T)=1$, we have $R(T)=\operatorname{span}(\{x\})$, and hence for any $u \in H$,

$$
T(u)=\langle T(u), x\rangle x=\left\langle u, T^{*}(x)\right\rangle x=\langle u, y\rangle x=T_{x, y}(u) .
$$

Thus $T=T_{x, y}$.

We now proceed to prove the main theorem. Recall that for Hilbert spaces $H, K$, a linear map $\Phi: B L(H) \rightarrow B L(K)$ is called an isomorphism if it is one-one and $\Phi(T S)=\Phi(T) \Phi(S)$ for all $T, S \in B L(H)$. An isomorphism $\Phi$ is called a *-isomorphism if $\Phi\left(T^{*}\right)=(\Phi(T))^{*}$ for all $T \in B L(H)$.

Theorem 2.3. Let $H$ and $K$ be Hilbert spaces and let $\Phi$ be ${ }^{*}{ }^{*}$-isomorphism from $B L(H)$ onto $B L(K)$. Then there exists a unitary operator $U: H \rightarrow K$ such that $\Phi(T)=U T U^{*}$ for all $T \in B L(H)$. Further, this unitary operator $U$ is unique up to a scalar multiple of absolute value 1 , that is, if $U: H \rightarrow K$ and $V: H \rightarrow K$ are unitary operators satisfying $U T U^{*}=\Phi(T)=V T V^{*}$ for all $T \in B L(H)$, then there exists a scalar $\lambda$, such that $|\lambda|=1$ and $V=\lambda U$.

Proof. We divide the proof into five steps.

Step 1. For each $x \in H$ with $\|x\|=1$, there exists $\tilde{x} \in K$ with $\|\tilde{x}\|=1$ such that $\Phi\left(P_{x}\right)=P_{\tilde{x}}$.

Let $x \in H$ with $\|x\|=1$. By Proposition 2.2, $P_{x}$ is an orthogonal projection of rank 1. Then

$$
\left(\Phi\left(P_{x}\right)\right)^{2}=\Phi\left(\left(P_{x}\right)^{2}\right)=\Phi\left(P_{x}\right) .
$$


Hence $\Phi\left(P_{x}\right)$ is a projection. Also since

$$
\left(\Phi\left(P_{x}\right)\right)^{*}=\Phi\left(\left(P_{x}\right)^{*}\right)=\Phi\left(P_{x}\right),
$$

$\Phi\left(P_{x}\right)$ is a self-adjoint and hence orthogonal projection.

We claim that $\Phi\left(P_{x}\right)$ is of rank 1. If not, there exist $y, z \in R\left(\Phi\left(P_{x}\right)\right)$ such that $\|y\|=1=\|z\|$ and $\langle y, z\rangle=0$. Consider the orthogonal projections $P_{y}, P_{z}$ in $B L(K)$. Since

$$
R\left(P_{y}\right)=\operatorname{span}(\{y\}) \subseteq R\left(\Phi\left(P_{x}\right)\right),
$$

we have $P_{y} \Phi\left(P_{x}\right)=P_{y}$. Since $\Phi^{-1}$ is also a ${ }^{*}$-isomorphism, we have $\Phi^{-1}\left(P_{y}\right)$ is an orthogonal projection and $\Phi^{-1}\left(P_{y}\right) P_{x}=\Phi^{-1}\left(P_{y}\right)$. Hence

$$
R\left(\Phi^{-1}\left(P_{y}\right)\right) \subseteq R\left(P_{x}\right)
$$

Similarly,

$$
R\left(\Phi^{-1}\left(P_{z}\right)\right) \subseteq R\left(P_{x}\right) .
$$

Now there exist nonzero elements $u \in R\left(\Phi^{-1}\left(P_{y}\right)\right)$ and $v \in R\left(\Phi^{-1}\left(P_{z}\right)\right)$. Then $u, v \in R\left(P_{x}\right)=\operatorname{span}(\{x\})$. On the other hand, since $\langle y, z\rangle=0, P_{y} P_{z}=0$, so that $\Phi^{-1}\left(P_{y}\right) \Phi^{-1}\left(P_{z}\right)=0$. Thus

$$
R\left(\Phi^{-1}\left(P_{y}\right)\right) \perp R\left(\Phi^{-1}\left(P_{z}\right)\right),
$$

consequently, $u \perp v$, a contradiction, proving the claim. Thus $\Phi\left(P_{x}\right)$ is an orthogonal projection of rank 1 . Hence by Proposition 2.2 there exists $\tilde{x} \in K$ with $\|\tilde{x}\|=1$ such that $\Phi\left(P_{x}\right)=P_{\tilde{x}}$.

Step 2. The construction of the unitary map $U$.

Fix $x_{0} \in H$ with $\left\|x_{0}\right\|=1$. Using Step 1 , choose $\tilde{x}_{0} \in K$ with $\left\|\tilde{x}_{0}\right\|=1$ such that $\Phi\left(P_{x_{0}}\right)=P_{\tilde{x}_{0}}$. Now define $U: H \rightarrow K$ by

$$
U(y):=\Phi\left(T_{y, x_{0}}\right)\left(\tilde{x}_{0}\right), \quad y \in H .
$$

Note that

$$
U\left(x_{0}\right):=\Phi\left(T_{x_{0}, x_{0}}\right)\left(\tilde{x}_{0}\right)=\Phi\left(P_{x_{0}}\right)\left(\tilde{x}_{0}\right)=P_{\tilde{x}_{0}}\left(\tilde{x}_{0}\right)=\tilde{x}_{0} .
$$

Clearly, $U$ is linear by Proposition 2.2. Now to prove that $U$ is unitary, consider $y, z \in H$. Then,

$$
\begin{aligned}
\langle U(y), U(z)\rangle & =\left\langle\Phi\left(T_{y, x_{0}}\right)\left(\tilde{x}_{0}\right), \Phi\left(T_{z, x_{0}}\right)\left(\tilde{x}_{0}\right)\right\rangle \\
& =\left\langle\left(\Phi\left(T_{z, x_{0}}\right)\right)^{*} \Phi\left(T_{y, x_{0}}\right)\left(\tilde{x}_{0}\right), \tilde{x}_{0}\right\rangle \\
& =\left\langle\Phi\left(\left(T_{z, x_{0}}\right)^{*}\right) \Phi\left(T_{y, x_{0}}\right)\left(\tilde{x}_{0}\right), \tilde{x}_{0}\right\rangle \\
& =\left\langle\Phi\left(T_{x_{0}, z} T_{y, x_{0}}\right)\left(\tilde{x}_{0}\right), \tilde{x}_{0}\right\rangle \\
& =\left\langle\Phi\left(\langle y, z\rangle P_{x_{0}}\right)\left(\tilde{x}_{0}\right), \tilde{x}_{0}\right\rangle \\
& =\langle y, z\rangle\left\langle P_{\tilde{x}_{0}}\left(\tilde{x}_{0}\right), \tilde{x}_{0}\right\rangle \\
& =\langle y, z\rangle\left\langle\tilde{x}_{0}, \tilde{x}_{0}\right\rangle=\langle y, z\rangle .
\end{aligned}
$$

This proves that $U$ preserves inner products. Next we prove that $U$ is onto. This will imply that $U$ is unitary. For this, let $v \in K$ and consider

$$
y:=\left(\Phi^{-1}\left(T_{v, \tilde{x_{0}}}\right)\right)\left(x_{0}\right) .
$$


Then, for $u \in H$,

$$
\begin{aligned}
T_{y, x_{0}}(u) & =\left\langle u, x_{0}\right\rangle y \\
& =\left\langle u, x_{0}\right\rangle\left(\Phi^{-1}\left(T_{v, \tilde{x_{0}}}\right)\right)\left(x_{0}\right) \\
& =\left(\Phi^{-1}\left(T_{v, \tilde{x_{0}}}\right)\right) P_{x_{0}}(u) .
\end{aligned}
$$

Hence,

$$
T_{y, x_{0}}=\Phi^{-1}\left(T_{v, \tilde{x_{0}}}\right) P_{x_{0}} .
$$

Thus,

$$
\Phi\left(T_{y, x_{0}}\right)=T_{v, \tilde{x_{0}}} \Phi\left(P_{x_{0}}\right)=T_{v, \tilde{x_{0}}} P_{\tilde{x_{0}}}=T_{v, \tilde{x_{0}}} .
$$

The last equality follows from Proposition 2.2(8). Now

$$
U(y):=\Phi\left(T_{y, x_{0}}\right)\left(\tilde{x}_{0}\right)=T_{v, \tilde{x_{0}}}\left(\tilde{x_{0}}\right)=\left\langle\tilde{x_{0}}, \tilde{x_{0}}\right\rangle v=v .
$$

This proves that $U$ is onto and hence unitary.

Step 3. $\Phi(T)=U T U^{*}$ for all $T \in B L(H)$ with $\operatorname{Rank}(T)=1$.

First we prove this for $T=T_{x_{0}, y}$ for $y \in H$. So, let $y \in H$. Then, for all $u \in H$,

$$
\begin{aligned}
\Phi\left(T_{x_{0}, y}\right)(U(u)) & =\Phi\left(T_{x_{0}, y}\right) \Phi\left(T_{u, x_{0}}\right)\left(\tilde{x}_{0}\right) \\
& =\Phi\left(T_{x_{0}, y} T_{u, x_{0}}\right)\left(\tilde{x}_{0}\right)=\Phi\left(\langle u, y\rangle P_{x_{0}}\right)\left(\tilde{x}_{0}\right) \\
& =\langle u, y\rangle \Phi\left(P_{x_{0}}\right)\left(\tilde{x}_{0}\right) \\
& =\langle u, y\rangle P_{\tilde{x}_{0}}\left(\tilde{x}_{0}\right) \\
& =\langle u, y\rangle \tilde{x}_{0} \\
& =\langle u, y\rangle U\left(x_{0}\right) \\
& =U\left(\langle u, y\rangle x_{0}\right) \\
& =U T_{x_{0}, y}(u) .
\end{aligned}
$$

Thus $\Phi\left(T_{x_{0}, y}\right) U=U T_{x_{0}, y}$, that is, $\Phi\left(T_{x_{0}, y}\right)=U T_{x_{0}, y} U^{*}$.

Next, let $T \in B L(H)$ with $\operatorname{Rank}(T)=1$. By Proposition 2.2(9), there exist $x, y \in H$ with $\|x\|=1$ such that $T=T_{x, y}$. But,

$$
T_{x, y}=T_{x, x_{0}} T_{x_{0}, y}=\left(T_{x_{0}, x}\right)^{*} T_{x_{0}, y} .
$$

Hence

$$
\begin{aligned}
\Phi(T) & =\left(\Phi\left(T_{x_{0}, x}\right)\right)^{*} \Phi\left(T_{x_{0}, y}\right) \\
& =\left(U T_{x_{0}, x} U^{*}\right)^{*} U T_{x_{0}, y} U^{*} \\
& =U\left(T_{x_{0}, x}\right)^{*} U^{*} U T_{x_{0}, y} U^{*} \\
& =U T_{x, x_{0}} T_{x_{0}, y} U^{*} \\
& =U T_{x, y} U^{*} \\
& =U T U^{*} .
\end{aligned}
$$

Step 4. $\Phi(T)=U T U^{*}$ for all $T \in B L(H)$.

Let $T \in B L(H)$ and let $x \in H$ with $\|x\|=1$. Then $\operatorname{Rank}\left(T P_{x}\right)$ is 0 or 1 . Hence by Step 3 ,

$$
U T P_{x} U^{*}=\Phi\left(T P_{x}\right)=\Phi(T) \Phi\left(P_{x}\right)=\Phi(T) U P_{x} U^{*} .
$$

Now evaluating both sides of the above equation at $U(x)$ and observing that $U^{*} U(x)=x$ and $P_{x}(x)=x$, we get $U T(x)=\Phi(T) U(x)$. Since this holds for all $x \in H$ with $\|x\|=1$, we have $U T=\Phi(T) U$, that is, $\Phi(T)=U T U^{*}$. 
Step 5. Uniqueness of $U$.

Suppose $U: H \rightarrow K$ and $V: H \rightarrow K$ are unitary operators satisfying $U T U^{*}=$ $\Phi(T)=V T V^{*}$ for all $T \in B L(H)$. Then we note that for all $y \in H, y=T_{y, x_{0}}\left(x_{0}\right)$. Hence

$$
\begin{aligned}
V(y) & =V T_{y, x_{0}}\left(x_{0}\right) \\
& =V T_{y, x_{0}} V^{*} V\left(x_{0}\right) \\
& =U T_{y, x_{0}} U^{*} V\left(x_{0}\right) \\
& =U\left(\left\langle U^{*} V\left(x_{0}\right), x_{0}\right\rangle y\right) \\
& =\left\langle U^{*} V\left(x_{0}\right), x_{0}\right\rangle U(y) .
\end{aligned}
$$

Thus $V=\lambda U$, where $\lambda=\left\langle U^{*} V\left(x_{0}\right), x_{0}\right\rangle=\left\langle V\left(x_{0}\right), U\left(x_{0}\right)\right\rangle$. Since $U$ and $V$ are unitary, $|\lambda|=1$. This completes the proof of the theorem.

Remark 2.4. A careful examination of the proof of Theorem 2.3 shows that the proof works even if we replace $B L(H)$ by a ${ }^{*}$-subalgebra $\mathcal{A} \subseteq B L(H)$ and $B L(K)$ by a ${ }^{*}$-subalgebra $\mathcal{B} \subseteq B L(K)$ such that $\mathcal{A}$ and $\mathcal{B}$ contain all operators of rank 1 . In particular, we can take $\mathcal{A}=C L(H)$, the algebra of all compact operators on $H$, and $\mathcal{B}=C L(K)$, the algebra of all compact operators on $K$. In fact, in [1] this theorem is first proved for the *-isomorphism of $C L(H)$ and then extended to the case of the *-isomorphism of $B L(H)$.

Remark 2.5. Note that we have not used the continuity of $\Phi$ in our proof of Theorem 2.3. It is a consequence of Theorem 2.3. The usual proof of this fact involves spectral considerations, whereas our proof does not.

\section{REFERENCES}

[1] W. Arveson, An Invitation to $C^{*}$-Algebra, Springer-Verlag, 1976. MR0512360 (58:23621)

[2] B. V. Limaye, Functional Analysis, New Age International, 1996. MR1427262 (97k:46001)

[3] A. E. Taylor and D.C. Lay, Introduction to Functional Analysis, John Wiley, 1980. MR0564653 (81b:46001)

Department of Mathematics, Indian Institute of Technology - Madras, Chennai 600036, INDIA

E-mail address: shk@iitm.ac.in

Department of Mathematics, Indian Institute of Technology - Madras, Chennai 600036, INDIA

E-mail address: mtnair@iitm.ac.in

Department of Mathematics, Cochin University of Science and Technology, Kochi682002 , INDIA

E-mail address: nambu@cusat.ac.in 